\title{
SOLUCION ESTADÍSTICA PARA UNA ANOMALIA EN EL CÁLCULO DE LA ENERGÍA INTERNA DE UN SISTEMA COMPUESTO, EN EL CONTEXTO DE LA MECÁNICA ESTADÍSTICA NO-EXTENSIVA
}

\author{
Felipe Américo Reyes Navarro ${ }^{\mathrm{a}^{*}}$, Jaime Francisco Vento Flores ${ }^{\mathrm{a}}$ \\ ${ }^{a}$ Facultad de Ciencias Físicas, Universidad Nacional Mayor de San Marcos Ap. Postal 14-0149, Lima 14, Perú.
}

(Recibido Junio 11, 2009; Aceptado Setiembre 19, 2009)

\begin{abstract}
Resumen
En este artículo, en el contexto de la $3^{\mathrm{a}}$ versión de la mecánica estadística no extensiva, teoría que se presenta como una generalización de la estadística padrón de Boltzmann-Gibbs-Shannon, presentamos una solución a una anomalía encontrada en el cálculo de la energía interna para un sistema compuesto $\mathrm{A}+\mathrm{B}$, de 2 spines $1 / 2$ de Hamiltoniano aditivo $H=H_{A}+H_{B}$, específicamente, el cálculo de la energía interna en el espacio de Hilbert completo es diferente al cálculo realizado en los subespacios de Hilbert, en otras palabras, $U \neq U_{A}+U_{B}$. Realizamos tanto cálculos analíticos (para 2 spines $1 / 2$ ), como simulaciones computaciones (para spines $S_{A}=2$ y $S_{B}=3 / 2$ ). Los resultados indican, de manera exacta, que el método alternativo de las matrices $E_{A}$ y $E_{B}$ es el indicado para los cálculos de la energía interna, por consiguiente, la matriz que contiene la información física del sistema es la matriz $\rho^{q}$ y no la matriz $\rho$, como si es el caso de la estadística padrón.
\end{abstract}

PACS: 78.66.Sq, 05.30.-d, 98.38.Am, 75.10.Dg

Palabras claves: mecánica estadística cuántica, sistema compuesto, propiedades físicas, Hamiltonianos de spin, energía interna y magnetización.

\begin{abstract}
Therein, inside of $3^{\text {rd }}$. version of nonextensive statistical mechanics, a generalization of the standard Boltzmann-Gibbs-Shannon statistics, we display a solution to an anomaly found in the calculation of internal energy for a composite A+B, 2 spins $1 / 2$ with additive Hamiltonian $H=H_{A}+H_{B}$, specifically, by calculating internal energy in full Hilbert space is different to calculating it into the Hilbert subspaces, in other words, $U \neq$ $U A+U B$. We make as much analytical calculations (for 2 spins $1 / 2$ ), as computational simulations (for spins $S_{A}=2$ and $S_{B}=3 / 2$ ). The results indicate, of exact way, that the alternative method of matrices $E_{A}$ and $E_{B}$ is exact for the calculations with internal energy, therefore, $\rho^{q}$ is the matrix that contains the physical information of the system but not the matrix $\rho$, as happen in the standard statistical.
\end{abstract}

Keywords: quantum statistical mechanics, composites, physical properties, spin Hamiltonian, internal energy and magnetization.

\section{Introducción}

La motivación para este trabajo son las investigaciones que han sido desarrolladas en los últimos 20 años utilizando la llamada mecánica estadística no-extensiva [1], teoría desarrollada por el investigador brasileño $\mathrm{C}$. Tsallis, que se presenta como una alternativa para la estadística de Boltzmann-Gibbs-Shannon. La cantidad de trabajos científicos a respecto de esta teoría es elevadísima, habiendo hasta la fecha más de 2000 publicaciones [2]. Estos estudios han abarcado las disciplinas más variadas desde teorías de campo hasta la física de la materia condensada; dentro del marco de esa teoría, analizamos un problema encontrado en la energía interna (en ese contexto también llamada $q$-energía interna): los cálculos del mencionado potencial termodinámico en diferentes espacios de Hilbert producen resultado distintos. La solución para el problema mencionado de la energía interna tuvo su origen en el artículo $A$ study on Composed Nonextensive Magnetic Systems [3], una ampliación de ese estudio puede ser visto en [4].

\footnotetext{
*Correspondig author. e-mail: jventof@unmsm.edu.pe and farnape@gmail.com
} 


\section{Marco Teórico}

La construcción de la mecánica estadística noextensiva comienza con el postulado de la entropía de Tsallis $S_{q}$ [2]:

$S_{q}=k_{B} \frac{1-\operatorname{Tr}\left(\hat{\rho}^{q}\right)}{q-1}$,

donde $\hat{\rho}^{q}$ es el operador matriz densidad $\hat{\rho}$ elevado a la $q$ potencia, siendo $q$ el índice entrópico y $k_{B}$ la constante de Boltzmann. Tr simboliza la operación de traza parcial. En el límite cuando $q$ tiende al valor de 1 recuperamos la entropía de Boltzmann-Gibbs.

$S=k_{B} \operatorname{Tr}(\hat{\rho} \operatorname{Ln} \hat{\rho})$,

El siguiente paso es encontrar la distribución de probabilidad que maximice la entropía de la Eq. (1), (debemos resaltar el hecho que se postule la entropía sin conocer a priori la matriz densidad). Esto es realizado a través del método de máxima entropía, considerando ciertas ligaduras para el sistema en cuestión. Este método considera a la mecánica estadística como un proceso de inferencia y fue inventado por el estadounidense Edward T. Jaynes en 1957.

Para un sistema compuesto $\mathrm{A}+\mathrm{B}$, tenemos la entropía del sistema completo:

$S_{A+B}=k_{B} \frac{1-\operatorname{Tr}_{(A, B)}\left[\hat{\rho}^{q}\right]}{q-1}$,

en tanto que para los subsistemas A y B, tenemos:

$S_{A}=k_{B} \frac{1-\operatorname{Tr}_{A}\left(\hat{\rho}_{A}^{q}\right)}{q-1}$ y $S_{B}=k_{B} \frac{1-\operatorname{Tr}_{B}\left(\hat{\rho}_{B}^{q}\right)}{q-1}$,

estas entropías para los subsistemas también son postuladas.

Actualmente, existen 3 versiones en el contexto de la mecánica estadística no-extensiva. Todas usan la misma forma entrópica de la Eq. (1). Sin embargo, es la $3^{\mathrm{a}}$ versión la más aceptada y ampliamente investigada por la comunidad científica. Esta última versión fue ideada por C. Tsallis, A. R. Plastino y R. $\mathrm{S}$. Mendes en 1998 en el artículo The Role of Constraints within Generalized Nonextensive Statistics [3]. Las ligaduras a ser consideradas, respectivamente, son las de norma unitaria y una redefinición de la energía interna:
$\operatorname{Tr}(\hat{\rho})=1 \quad$ y $\quad U_{q}=\frac{\operatorname{Tr}\left(\hat{\rho}^{q} \hat{H}\right)}{\operatorname{Tr}\left(\hat{\rho}^{q}\right)}$,

encontrándose, después del proceso de maximización de $S_{q}$, la siguiente densidad de probabilidad:

$\hat{\rho}=\frac{\left[\hat{1}-(1-q) \beta^{\prime} \hat{H}\right]^{\frac{1}{1-q}}}{Z_{q}}$,

$Z_{q}$ es la función de partición,

$Z_{q}=\operatorname{Tr}\left[\hat{1}-(1-q) \beta^{\prime} \hat{H}\right]^{\frac{1}{1-q}}$,

donde $\beta^{\prime}$ es un parámetro energético, ver los trabajos de M. S. Reis et al. [4, 5, 6, 7].

\section{Procedimiento}

El sistema de estudio es un sistema magnético de 2 spines $1 / 2$, de Hamiltoniano $H=H_{A}+H_{B}$, usamos el modelo de Heisenberg en la aproximación de campo medio. Los observables físicos serán representados por operadores, así el sistema de estudio se expresa:

$\hat{H}_{A+B}=\hat{H}_{A}+\hat{H}_{B}$,

Siendo

$$
\begin{aligned}
& \hat{H}_{A}=g \mu_{h} B_{A} \sum_{i}^{N} \hat{S}_{A, i}^{z} \quad \mathrm{y} \\
& \hat{H}_{B}=g \mu_{h} B_{B} \sum_{i}^{N} \hat{S}_{B, i}^{z},
\end{aligned}
$$

$g=2$ es el factor giromagnético, $\mu_{h}$ es el magnetón de Bohr, por razones didácticas cambiamos el símbolo usual $\mu_{B} ; N_{A}$ y $N_{B}$ son los respectivos números de iones de las subredes A y B; $\hat{S}_{A, i}^{z}$ y $\hat{S}_{B, i}^{z}$ son los operadores de spin, $\mathrm{A}(\mathrm{B})$ indica la respectiva subrrede y el subíndice $i$, la i-ésima partícula; $B_{A}$ y $B_{B}$ son los llamados campos magnéticos efectivos, definidos como:

$$
\begin{aligned}
& B_{A}=B_{0}+\lambda_{A} M_{A}+\lambda_{A B} M_{B} \quad \mathrm{y} \\
& B_{B}=B_{0}+\lambda_{B} M_{B}+\lambda_{A B} M_{A},
\end{aligned}
$$

donde $\mathrm{B}_{0}$ es el campo magnético externo aplicado, $\lambda_{\mathrm{A}}$ y $\lambda_{\mathrm{B}}$ son parámetros de acoplamiento intrared y 
$\lambda_{\mathrm{AB}}$, es el parámetro de acoplamiento interred. Considerando que los spines son independientes la Eq. (9) se expresa como:

$$
\begin{aligned}
& \hat{H}_{A}=g \mu_{h} B_{A} N_{A} \hat{S}_{A}^{z} \quad \mathrm{y} \\
& \hat{H}_{B}=g \mu_{h} B_{B} N_{B} \hat{S}_{B}^{z} .
\end{aligned}
$$

En las siguientes subsecciones mostramos los cálculos realizados para encontrar la energía interna.

\subsection{Cálculo de las Energías Internas en el Espacio de Hilbert Completo $4 x 4$}

En esta subsección mostramos cómo se aplica la mecánica estadística no-extensiva, para el sistema magnético representado por la Eq. (9), considerando $N_{A}=N_{B}=N$ para una simplificación de los cálculos. En el espacio de Hilbert completo, las energías internas tienen las fórmulas generales:

$$
\begin{aligned}
U_{A} & =\frac{\operatorname{Tr}_{(A, B)}\left(\hat{\rho}^{q} \hat{H}_{A}\right)}{\operatorname{Tr}_{(A, B)}\left(\hat{\rho}^{q}\right)} \quad \mathrm{y} \\
U_{B} & =\frac{\operatorname{Tr}_{(A, B)}\left(\hat{\rho}^{q} \hat{H}_{A}\right)}{\operatorname{Tr}_{(A, B)}\left(\hat{\rho}^{q}\right)}
\end{aligned}
$$

así, para nuestro sistema específico de 2 spines $1 / 2$., en un espacio de Hilbert $4 \times 4$, las respectivas energías internas, en un espacio de Hilbert $4 \times 4$, adoptan la forma:

$$
\begin{aligned}
& U_{A}=N g \mu_{h} B_{A} \frac{\operatorname{Tr}_{(A, B)}\left(\hat{\rho}^{q} \hat{S}_{A}^{z}\right)}{\operatorname{Tr}_{(A, B)}\left(\hat{\rho}^{q}\right)} \quad \mathrm{y} \\
& U_{B}=N g \mu_{h} B_{B} \frac{\operatorname{Tr}_{(A, B)}\left(\hat{\rho}^{q} \hat{S}_{B}^{z}\right)}{\operatorname{Tr}_{(A, B)}\left(\hat{\rho}^{q}\right)}
\end{aligned}
$$

Para calcular estos parámetros, necesitaremos la matriz densidad, así como los operadores de spin. Primero, de la Eq. (6) formamos el operador matriz densidad:

$\hat{\rho}=\left\{1+(1-q) \frac{2 N \mu_{h}}{K_{B} T}\left(\hat{S}_{A}^{z} B_{A}+\hat{S}_{B}^{z} B_{B}\right\}^{\frac{1}{1-q}} / Z_{q}\right.$

el cual elevamos a la potencia $q$ :

$$
\hat{\rho}^{q}=\left\{1+(1-q) \frac{2 N \mu_{h}}{k_{B} T}\left(S_{A}^{z} B_{A}+S_{B}^{z} B_{B}\right\}^{\frac{q}{1-q}} / Z_{q}^{q}\right.
$$

Por otro lado, los operadores adimensionales de spin en el espacio de Hilbert completo $4 \times 4$ son ( $\hbar$ está incluido en el magnetón de Bohr):

$\hat{S}_{A}^{z}=\{|++\rangle\langle++|+|+-\rangle\langle+-|-|-+\rangle\langle-+|-|--\rangle\langle--|\} / \quad \mathrm{y}$
$\hat{S}_{B}^{z}=\{|++\rangle\langle++|-|+-\rangle\langle+-|+|-+\rangle\langle-+|-|--\rangle\langle-|\} / 2$

donde $|++\rangle\langle++||+-\rangle,\langle+-||-+\rangle,\langle-+|\mathrm{y}|--\rangle\langle--|$ son operadores externos, los cuales son formados por bras y kets, los cuales representan estados cuánticos del sistema completo. Los bras son: $\langle++|,\langle+-|,\langle-+|$ y $\langle--| ;$ los respectivos kets son: $|++\rangle,|+-\rangle,|-+\rangle$ y $|--\rangle$. Ahora, podemos obtener los elementos matriciales de la matriz densidad:

$$
\begin{aligned}
& \rho_{++}=\langle++|\hat{\rho}|++\rangle=\alpha_{1} / Z_{q}, \\
& \rho_{+-}=\langle+-|\hat{\rho}|+-\rangle=\alpha_{2} / Z_{q}, \\
& \rho_{-+}=\langle-+|\hat{\rho}|-+\rangle=\alpha_{3} / Z_{q} \quad \mathrm{y} \\
& \rho_{--}=\langle--|\hat{\rho}|--\rangle=\alpha_{4} / Z_{q},
\end{aligned}
$$

donde por razones prácticas, hemos introducido los siguientes parámetros $\alpha$ :

$$
\begin{aligned}
& \alpha_{1}=\left[1+(1-q) \frac{2 N \mu_{h}\left(B_{A}+B_{B}\right)}{k_{B} T}\right]^{\frac{1}{1-q}}, \\
& \alpha_{2}=\left[1+(1-q) \frac{2 N \mu_{h}\left(B_{A}-B_{B}\right)}{k_{B} T}\right]^{\frac{1}{1-q}}, \\
& \alpha_{3}=\left[1+(1-q) \frac{2 N \mu_{h}\left(-B_{A}+B_{B}\right)}{k_{B} T}\right]^{\frac{1}{1-q}} \mathrm{y} \\
& \alpha_{4}=\left[1-(1-q) \frac{2 N \mu_{h}\left(B_{A}+B_{B}\right)}{k_{B} T}\right]^{\frac{1}{1-q}}
\end{aligned}
$$

y para los elementos matriciales de $\rho^{q}$ tenemos: 

\begin{tabular}{ll}
$\rho_{++}^{q}=\left\langle++\left|\hat{\rho}^{q}\right|++\right\rangle=\alpha_{1}^{q} / Z_{q}^{q}$, & $\rho_{--}^{q}=\left\langle--\left|\hat{\rho}^{q}\right|--\right\rangle$ \\
$\rho_{+-}^{q}=\left\langle+-\left|\hat{\rho}^{q}\right|+-\right\rangle=\alpha_{2}^{q} / Z_{q}^{q}$, & por lo tanto, mediant \\
el operador matriz & la siguiente manera: \\
$\rho_{-+}^{q}=\left\langle-+\left|\hat{\rho}^{q}\right|-+\right\rangle=\alpha_{3}^{q} / Z_{q}^{q} \quad \mathrm{y}$ & \\
\hline$\hat{\rho}=\left\{\alpha_{1}|++\rangle\left\langle++\left|+\alpha_{2}\right|+-\right\rangle\left\langle+-\left|+\alpha_{3}\right|-+\right\rangle\left\langle-+\left|+\alpha_{4}\right|--\right\rangle\langle--|\right\} / Z_{q}$
\end{tabular}

por lo tanto, mediante el uso de operadores externos, operador matriz densidad puede ser expresado de

y elevado a la potencia $q$ :

$\hat{\rho}^{q}=\left\{\alpha_{1}^{q}|++\rangle\left\langle++\left|+\alpha_{2}^{q}\right|+-\right\rangle\left\langle+-\left|+\alpha_{3}^{q}\right|-+\right\rangle\left\langle-+\left|+\alpha_{4}^{q}\right|--\right\rangle\langle--|\right\} / Z_{q}^{q}$

de esta manera, reemplazando las Eqs. (16) y (21) en la Eq.(13), obtenemos las energías internas en el espacio de Hilbert completo 4x4:

$U_{A}=N \mu_{h} B_{A} \frac{\alpha_{1}^{q}+\alpha_{2}^{q}-\alpha_{3}^{q}-\alpha_{4}^{q}}{\alpha_{1}^{q}+\alpha_{2}^{q}+\alpha_{3}^{q}+\alpha_{4}^{q}} \quad \mathrm{y}$
$U_{B}=N \mu_{h} B_{B} \frac{\alpha_{1}^{q}+\alpha_{3}^{q}-\alpha_{2}^{q}-\alpha_{4}^{q}}{\alpha_{1}^{q}+\alpha_{2}^{q}+\alpha_{3}^{q}+\alpha_{4}^{q}}$

así, tenemos que la energía interna total es:

$U_{A+B}=N g \mu_{h} \frac{B_{A}\left\{\alpha_{1}^{q}+\alpha_{2}^{q}-\alpha_{3}^{q}-\alpha_{4}^{q}\right\}+B_{B}\left\{\alpha_{1}^{q}+\alpha_{3}^{q}-\alpha_{2}^{q}-\alpha_{4}^{q}\right\}}{\alpha_{1}^{q}+\alpha_{2}^{q}+\alpha_{3}^{q}+\alpha_{4}^{q}}$.

En las próximas dos subsecciones, utilizando dos procedimientos, estos resultados serán comparados con los cálculos en los subespacios de Hilbert 2x2.

\subsection{Cálculo de las Energías Internas en los Subespacios de Hilbert 2x2, Usando las Fórmulas Acostumbradas de la Tercera Versión}

Realizaremos el cálculo de $U_{A}$ y $U_{B}$ en los subespacios de Hilbert 2x2. En principio los resultados deben ser idénticos a aquellos obtenidos en el espacio de Hilbert $4 \times 4$. En los subespacios de Hilbert $2 \times 2$ las energías internas para el sistema de la Eq. (9) son definidas como:

$$
\begin{aligned}
& U_{A}=N g \mu_{h} B_{A} \frac{\operatorname{Tr}_{A}\left(\hat{\rho}_{A}^{q} \hat{s}_{A}^{z}\right)}{\operatorname{Tr}_{A}\left(\hat{\rho}_{A}^{q}\right)} \quad \mathrm{y} \\
& U_{B}=N g \mu_{h} B_{B} \frac{\operatorname{Tr}_{B}\left(\hat{\rho}_{B}^{q} \hat{s}_{B}^{z}\right)}{\operatorname{Tr}_{B}\left(\hat{\rho}_{B}^{q}\right)}
\end{aligned}
$$

para calcular estos parámetros termodinámicos, en la Eq. (20) realizamos la traza parcial y obtenemos las respectivas matrices parciales $\hat{\rho}_{A} \mathrm{y} \hat{\rho}_{B}:\{\mathrm{XE}$ "matrices parciales"

$$
\begin{aligned}
& \hat{\rho}_{A}=\left\{\left(\alpha_{1}+\alpha_{2}\right)|+\rangle\left\langle+\left|+\left(\alpha_{3}+\alpha_{4}\right)\right|-\right\rangle\langle-|\right\} / Z_{q} \quad \mathrm{y} \\
& \hat{\rho}_{B}=\left\{\left(\alpha_{1}+\alpha_{3}\right)|+\rangle\left\langle+\left|+\left(\alpha_{2}+\alpha_{4}\right)\right|-\right\rangle-\mid\right\} / Z_{q}
\end{aligned}
$$

elevando a la potencia q, tenemos:

$$
\begin{aligned}
& \hat{\rho}_{A}^{q}=\left\{\left(\alpha_{1}+\alpha_{2}\right)^{q}|+\rangle\left\langle+\left|+\left(\alpha_{3}+\alpha_{4}\right)^{q}\right|-\right\rangle\langle-|\right\} / Z_{q}^{q} \mathrm{y} \\
& \hat{\rho}_{B}^{q}=\left\{\left(\alpha_{1}+\alpha_{3}\right)^{q}|+\rangle\left\langle+\left|+\left(\alpha_{2}+\alpha_{4}\right)^{q}\right|-\right\rangle\langle-|\right\} / Z_{q}^{q}
\end{aligned}
$$

Para nuestro cálculo, también sabemos que los operadores de spin en los subespacios de Hilbert 2x2 son: 


$$
\begin{aligned}
& \hat{s}_{A}^{z}=\{|+\rangle\langle+|-|-\rangle\langle-|\} / 2 \quad \mathrm{y} \\
& \hat{s}_{B}^{z}=\{|+\rangle\langle+|-|-\rangle\langle-|\} / 2
\end{aligned}
$$

así, remplazando las Eqs. (26) y (27) en la Eq. (24), obtenemos el siguiente resultado en los respectivos espacios de Hilbert 2x2:

$$
\begin{aligned}
& U_{A}=N g \mu_{h} B_{A} \frac{\left(\alpha_{1}+\alpha_{2}\right)^{q}-\left(\alpha_{3}+\alpha_{4}\right)^{q}}{\left(\alpha_{1}+\alpha_{2}\right)^{q}+\left(\alpha_{3}+\alpha_{4}\right)^{q}} \quad \mathrm{y} \\
& U_{B}=N \mu_{h} B_{A} \frac{\left(\alpha_{1}+\alpha_{3}\right)^{q}-\left(\alpha_{2}+\alpha_{4}\right)^{q}}{\left(\alpha_{1}+\alpha_{2}\right)^{q}+\left(\alpha_{3}+\alpha_{4}\right)^{q}},
\end{aligned}
$$

de manera que la energía interna total es:

$$
U_{A+B}=N g \mu_{h} \frac{B_{A}\left\{\left(\alpha_{1}+\alpha_{2}\right)^{q}-\left(\alpha_{3}+\alpha_{4}\right)^{q}\right\}+B_{B}\left\{\left(\alpha_{1}+\alpha_{3}\right)^{q}-\left(\alpha_{2}+\alpha_{4}\right)^{q}\right\}}{\left(\alpha_{1}+\alpha_{2}\right)^{q}+\left(\alpha_{3}+\alpha_{4}\right)^{q}}
$$

notamos que estos resultados son diferentes al resultado conseguido en el espacio de Hilbert completo 4x4, Eqs. (22) y (23). Esto puede interpretarse como un resultado anómalo en el contexto de la mecánica estadística no-extensiva.

\subsection{Las Matrices $E_{A}$ y $E_{B}$ y el Cálculo Adecuado para las Energías Internas, en el Subespacio de Hilbert 2x2}

Para el sistema magnético representado por la Eq. (9), en esta subsección introducimos un procedimiento que fue dado a conocer en [6, 7]. Por razones didácticas, aquí llamamos de matrices $E_{A}$ y $E_{B}$ a las matrices $\rho_{A, q}$ y $\rho_{B, q}$ de la referencia [6]. Así, en este contexto, definimos las energías internas como,

$$
\begin{aligned}
U_{A} & =N g \mu_{h} B_{A} \frac{\operatorname{Tr}_{A}\left(\hat{E}_{A} \hat{s}_{A}^{z}\right)}{\operatorname{Tr}_{A}\left(\hat{E}_{A}\right)} \quad \mathrm{y} \\
U_{B} & =N g \mu_{h} B_{B} \frac{\operatorname{Tr}_{B}\left(\hat{E}_{B} \hat{s}_{B}^{z}\right)}{\operatorname{Tr}_{B}\left(\hat{E}_{B}\right)},
\end{aligned}
$$

donde las matrices parciales $E_{A}$ y $E_{B}$ son definidas como:

$$
\begin{aligned}
& E_{A}=\operatorname{Tr}_{B}\left(\rho^{q}\right) \quad \mathrm{y} \\
& E_{B}=\operatorname{Tr}_{A}\left(\rho^{q}\right)
\end{aligned}
$$

entonces, realizamos la traza parcial de la matriz $\rho^{q}$ en la Eq. (21):

$$
\begin{aligned}
& \hat{E}_{A}=\left\{\left(\alpha_{1}^{q}+\alpha_{2}^{q}\right)|++\rangle\left\langle++\left|+\left(\alpha_{3}^{q}+\alpha_{4}^{q}\right)\right|--\right\rangle\langle--|\right\} / Z_{q}^{q} \mathrm{y} \\
& \hat{E}_{B}=\left\{\left(\alpha_{1}^{q}+\alpha_{3}^{q}\right)|++\rangle\left\langle++\left|+\left(\alpha_{2}^{q}+\alpha_{4}^{q}\right)\right|--\right\rangle\langle--|\right\} / Z_{q}^{q}
\end{aligned}
$$

debemos resaltar que estas matrices ya no necesitan ser elevadas a la potencia $q$. Por lo tanto, remplazando las Eqs. (27) y (32) en la Eq. (30), encontramos que el uso de las matrices $E_{A}$ y $E_{B}$ produce las siguientes energías, en los subespacios de Hilbert 2x2:

$$
\begin{aligned}
& U_{A}=N g \mu_{h} B_{A} \frac{\alpha_{1}^{q}+\alpha_{2}^{q}-\alpha_{3}^{q}-\alpha_{4}^{q}}{\alpha_{1}^{q}+\alpha_{2}^{q}+\alpha_{3}^{q}+\alpha_{4}^{q}} \quad \mathrm{y} \\
& U_{B}=N g \mu_{h} B_{B} \frac{\alpha_{1}^{q}+\alpha_{2}^{q}-\alpha_{3}^{q}-\alpha_{4}^{q}}{\alpha_{1}^{q}+\alpha_{2}^{q}+\alpha_{3}^{q}+\alpha_{4}^{q}}
\end{aligned}
$$

en consecuencia la energía total del sistema $\mathrm{A}+\mathrm{B}$ es:

$U_{A+B}=N g \mu_{h} \frac{B_{A}\left\{\alpha_{1}^{q}+\alpha_{2}^{q}-\alpha_{3}^{q}-\alpha_{4}^{q}\right\}+B_{B}\left\{\alpha_{1}^{q}+\alpha_{3}^{q}-\alpha_{2}^{q}-\alpha_{4}^{q}\right\}}{\alpha_{1}^{q}+\alpha_{2}^{q}+\alpha_{3}^{q}+\alpha_{4}^{q}}$ 
estos resultados concuerdan exactamente con las Eqs. (22) y (23), calculadas en el espacio de Hilbert completo $4 \times 4$. Este hecho muestra de manera clara que las matrices parciales $E_{A}$ y $E_{B}$ son las adecuadas para el cálculo de la energía interna, en el contexto de la $3^{\text {a }}$ versión de la mecánica estadística no-extensiva

\section{Simulaciones Computacionales}

Los resultados analíticos anteriores pueden ser extendidos para otros valores de spin, p. e., $S_{A}=2$ y $S_{B}=3 / 2$, sin embargo, la dimensión del espacio de Hilbert completo es $20 \times 20=400$, pues $[2(3 / 2)+1][2(2)+1]=20$, por consiguiente para este caso es más práctico mostrar simulaciones computacionales que cálculos analíticos, estas simulaciones fueron realizadas con el lenguaje de programación Matlab. Los resultados confirman, una vez más, que las matrices parciales $E_{A}$ y $E_{B}$ son las idóneas. En la Fig. 1, para un sistema con spines $S_{A}=2$ y $S_{B}=3 / 2$, mostramos las gráficas de la Energía interna total $v s$. Temperatura, $\mathrm{U}_{\text {tot. }} v s$. T, (a) usando la $3^{\text {a }}$ versión de la mecánica estadística no-extensiva, en el espacio completo de Hilbert, asi como en los respectivos subespacios y (b) usando las fórmulas de $3^{\mathrm{a}}$ versión (sólamente en el espacio de Hilbert completo), así como el método de las matrices $E_{A}$ y $E_{B}$, en los respectivos subespacios de Hilbert. El modelo utilizado es idéntico al usado para el sistema de 2 spines 1/2. Adicionalmente, para el sistema de 2 spins $1 / 2$, para el caso antiferromagnético mostramos que para el calculo de la magnetización, el método de las matrices $E_{A}$ y $E_{B}$, también se confirma.

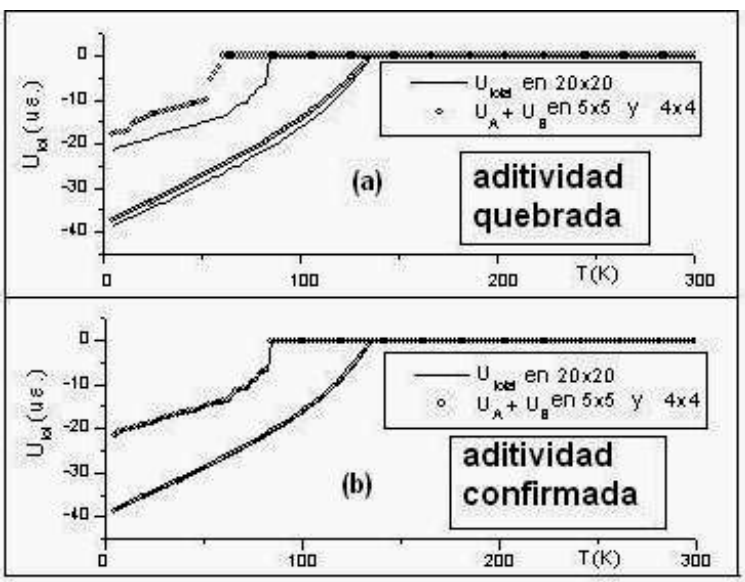

Fig. 1. Energía interna total vs. Temperatura, $\mathrm{U}_{\text {tot }} v s$. $\mathrm{T}$, para un sistema de spins $S_{A}=2$ y $S_{B}=3 / 2$. (a) usando la $3^{\text {a }}$ versión de la mecánica estadística no-extensiva, en el espacio de Hilbert 20x20 y en los respectivos subespacios de Hilbert $5 \times 5$ y $4 \times 4$, (b) usando las fórmulas de la $3^{\mathrm{a}}$ versión únicamente en el espacio de Hilbert 20x20, así como las fórmulas de las matrices $E_{A}$ y $E_{B}$ en los respectivos subespacios de Hilbert $5 \times 5$ y $4 \times 4$. Los valores de $q$ son, de la energía nula para abajo, 0.3 y 0.7 .

\section{Conclusiones}

En este trabajo hemos mostrado explícitamente que ocurre una anomalía en el cálculo de la energía interna de un sistema compuesto $\mathrm{A}+\mathrm{B}$, cuando se usan las fórmulas conocidas de la $3^{\mathrm{a}}$ versión de la mecánica estadística no-extensiva, en los subespacios de Hilbert. De igual manera, demostramos, de manera exacta, que la solución a ese problema es la introducción de las matrices $E_{A}$ y $E_{B}$, son estas matrices que contienen la información física de los subsistemas. Por lo tanto la operación de traza parcial se debe realizar sobre la matriz $\rho^{q}$ y no sobre la matriz $\rho$. Finalmente, la propuesta del método de las matrices $E_{A}$ y $E_{B}$ es confirmada tanto de manera analítica, de forma exacta, así como con simulaciones computacionales.

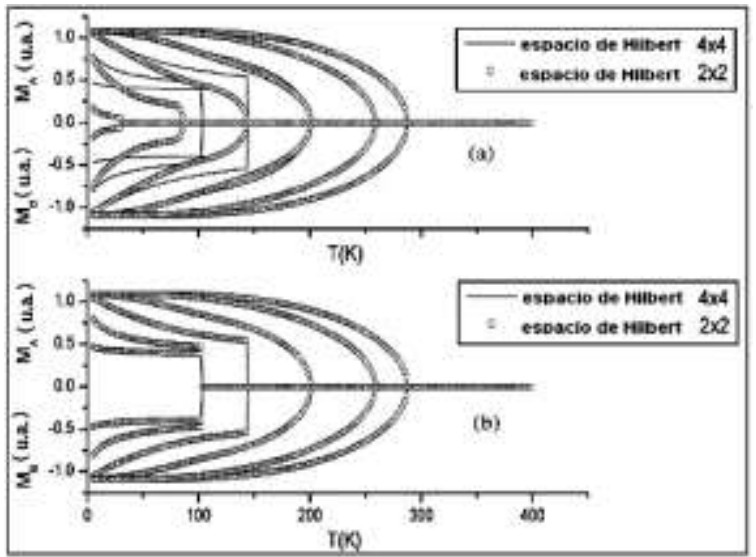

Fig. 2. Magnetizacionl vs. Temperatura, $U_{\text {tot }}$ vs. T, para un sistema antiferromagnético de 2 spins $S_{A}=1 / 2$ y $S_{B}=1 / 2$. (a) usando la $3^{\mathrm{a}}$. versión de la mecánica estadística noextensiva, en el espacio de Hilbert $4 \mathrm{x} 4 \mathrm{y}$ en los respectivos subespacios de Hilbert 2x2 y 2x2, (b) usando las fórmulas de la $3^{\text {a }}$ versión únicamente en el espacio de Hilbert $4 \times 4$, así como las fórmulas de las matrices $E_{A}$ y $E_{B}$ en los respectivos subespacios de Hilbert $2 \times 2$ y $2 \times 2$. Los valores de $q$ son, del origen para afuera, $0.1,0.3,0.5,0.7,0.9$ y 1 .

\section{References}

[1]. http://tsallis.cat.cbpf.br/biblio.htm

[2]. C. Tsallis, Phys. D 193, 3 (2004), condmat/0403012.

[3]. Tsallis, R.S. Mendes y R. Plastino, Physica A 261, 534 (1998). 
[4]. M. S. Reis, J. C. C. Freitas, M. T. D. Orlando, E. K. Lenzi e I. S. Oliveira, Europhys. Lett. 58 (1), 42 (2002), cond-mat/0109061.

[5]. M. S. Reis, J. P. Araujo y V. S. Amaral, E. K. Lenzi e I. S. Oliveira, Phys. Rev. B 66, 134417(2002), cond-mat/0207245.

[6]. F. A. R. Navarro, M. S. Reis, E. K. Lenzi e I. S. Oliveira, Phys. A 343, 499 (2004).

[7]. F. A. R. Navarro, M. S. Reis, E. K Lenzi e I. S. Olivera; cond-mat/0701495 (2007).
[8]. Robert K. Niven, ArXiv:0908.0990 (2009).

[9]. E.T. Jaynes, Phys. Rev., 106, 620 (1957).

[10]. E.T. Jaynes, Lectures in Theoretical Physics 3: Statistical Physics, 181. Ed. K.W. Ford, Brandeis University (1963).

[11]. E.T. Jaynes, Probability Theory: The Logic of Science, Cambridge. Ed. G.L. Bretthorst (2003). 